Projets

de paysage

\section{Projets de paysage}

Revue scientifique sur la conception et l'aménagement de l'espace

$18 \mid 2018$

Paysage et didactique

\title{
Le sentir : au fondement du politique
}

Pour une considération de l'horizon et d'un sol commun

Sensing: At the foundation of Politics - Taking into Consideration the Horizon and a Common Ground

\section{Catherine Grout}

\section{(2) OpenEdition}

\section{Journals}

Édition électronique

URL : http://journals.openedition.org/paysage/1055

DOI : 10.4000/paysage. 1055

ISSN : 1969-6124

\section{Éditeur :}

École nationale supérieure du paysage de Versailles-Marseille, Institut national des sciences appliquées Centre Val de Loire - École de la nature et du paysage, École nationale supérieure d'architecture et de paysage de Bordeaux, École nationale supérieure d'architecture et de paysage de Lille, Agrocampus Angers

\section{Référence électronique}

Catherine Grout, «Le sentir : au fondement du politique », Projets de paysage [En ligne], 18 | 2018, mis en ligne le 08 juillet 2018, consulté le 28 novembre 2019. URL : http://journals.openedition.org/ paysage/1055; DOI : 10.4000/paysage.1055

Ce document a été généré automatiquement le 28 novembre 2019.

Projets de paysage 


\title{
Le sentir : au fondement du politique
}

\author{
Pour une considération de l'horizon et d'un sol commun \\ Sensing: At the foundation of Politics - Taking into Consideration the Horizon \\ and a Common Ground
}

Catherine Grout

1 Deux principaux niveaux de sens se croisent dans la pédagogie que je vais présenter ici. Le premier concerne la manière de faire partie du monde environnant pour transformer celui-ci, le deuxième correspond à une considération de l'horizon et du commun ${ }^{1}$ appelant des spatialisations et des modes de conception. L'un n'irait pas sans l'autre. Le fond sur lequel je les déploie correspond au sentir, tel qu'il fut étudié par le neuropsychiatre Erwin Straus. Mode pathique de relation du sujet vivant au monde environnant dans l'unité sentir-se-mouvoir, le sentir correspond ainsi à un mode d'être non réflexif, aux mouvements spontanés et aux sollicitations sensorimotrices. Pour le neuropsychiatre proche de la phénoménologie, nous oscillons dans le quotidien entre «l'espace du paysage» (celui du sentir quand nous sommes en communication immédiate avec le monde qui nous entoure) et «l'espace de la géographie » (celui des déterminations, qu'elles soient géographiques, géométriques, celles des calculs ou des positions). Mon postulat, qui porte toute ma pédagogie et ma recherche, est que le sentir étaye l'horizon politique d'un projet de paysage, autrement dit une présence située au monde (et donc à autrui et en relation avec un horizon) assure potentiellement un fondement incarné au politique. Avec le sentir et la notion de commun se précise un horizon de sens propre à orienter le projet de manière concrète. Après avoir donné quelques éléments de référence autour de l'expérience, je présenterai six moments pédagogiques clés afin de relier le sentir au politique. J'insisterai sur l'importance d'une présence à soi et au monde ainsi que du rapport au sol et à l'horizon pour éprouver et envisager un sol commun. 


\section{«Le corps et l'environnement font partie de la même expérience »}

2 Mon postulat s'est construit avec des travaux en phénoménologie, en analyse du mouvement, en psychomotricité et en philosophie politique ainsi qu'avec la rencontre avec des œuvres et des artistes. Dans son ouvrage intitulé La Tête aux pieds, Odile Rouquet (1991, p. 20) écrit que « le corps et l'environnement font partie de la même expérience ${ }^{2} »$. Cette indication est-elle si évidente ? Sans doute devons-nous la prendre en compte en ayant en mémoire Hannah Arendt. La philosophe a relevé que le mouvement d'aliénation du monde (ou acosmisme) correspondait à un des trois dangers du $\mathrm{XX}^{\mathrm{e}}$ siècle avec le totalitarisme et la société de consommation. Dès lors, si le "corps et l'environnement font partie de la même expérience ", il convient de les traiter ensemble et non séparément. Autrement dit, l'analyse d'un site de projet passe par une attention portée à l'expérience vécue de celui-ci : or, comment accéder à ce qui est ressenti par nous-mêmes et par autrui ? Comment qualifier, comment évoquer les qualités, les difficultés, les inconforts, les rencontres avec autrui ? Il s'agit aussi de se demander comment l'expérience devient matière pour le projet et, pour cela, de faire vivre par l'enseignement combien notre propre construction de la représentation et de la cognition se fonde sur notre expérience corporelle et sensorimotrice. L'espace mental se construisant à partir de celle-ci, pour concevoir un espace, il importe de savoir comment nous le percevons et surtout comment nous le vivons, nous-mêmes et autrui. L'historienne des villes, Françoise Choay, met ainsi l'accent sur « la disparition de notre investissement corporel dans l'espace concret ». Elle insiste sur trois points: le « retrait du corps en mouvement » est associé à « la désaffectation corrélative de l'espace local à l'échelle du corps humain » et il est «consacré » par «la multiplication des prothèses qui, des transports ultrarapides et des télécommunications aux images de synthèse, médiatisent notre rapport à l'espace naturel et bâti ${ }^{3}$ " (Choay, 2006, p. 104). Si l'on suit son développement et si l'on écoute les commentaires des enseignant.e.s de projet remarquant la difficulté qu'ont souvent les étudiant.e.s à rendre compte de leur expérience des espaces, comme s'ils (elles) ne s'y trouvaient pas, il importe de les aider à revendiquer un «investissement corporel dans l'espace concret " pour qu'ils (elles) s'ouvrent à une relation immédiate et directe à partir de leur " corps en mouvement ", et se rendent compte des diverses médiatisations et filtres visuels qui ont pu passer inaperçus et aller de soi. La présence corporelle, le contact, la relation sentir-semouvoir sont la base, avec la respiration, de tout le rapport au monde, à la volumétrie, aux interrelations, à la profondeur et à l'orientation. Sans une expérience vécue, considérée pour ce qu'elle est, et articulée au projet, celui-ci risque d'être désincarné, sans destination, «indifférent au devenir» (Straus, 1989, p. 622) des sujets vivants, voire sans pensée spatiale et temporelle. Comment accéder à cette matière associant présence corporelle et environnement, relation au monde et mouvements, comment la travailler, quels sont les outils? Comment aussi décentrer son approche (ne pas rester sur soi) pour avoir une relation à l'horizon, à un espace environnant plus ou moins partagé ? Comment destiner son projet à autrui ?

3 Dans mon enseignement à l'École nationale supérieure d'architecture et de paysage de Lille, je fais appel aux compétences venant de la danse et de la chorégraphie afin d'aider les étudiant.e.s à envisager leur expérience quotidienne comme étant située $e^{4}$, polysensorielle, en contact avec une certaine qualité de sol, d'atmosphère, en relation 
avec autrui et se déployant dans une profondeur associée à l'horizon. Mon ambition pédagogique est ainsi de permettre aux étudiant.e.s qu'ils (elles) puissent en parler et s'en saisir, pour que leur projet en soit nourri et informé (que des formes et mises en forme en découlent ${ }^{5}$ ). Il s'agit que chacun.e se situe dans (et avec) son corps de manière interne, de mobiliser celui-ci et de l'éprouver comme un volume, et donc de ne pas seulement le considérer comme un objet isolé, mais bien comme un échange permanent et en transformation, non séparé du monde environnant. À la suite d'un laboratoire avec la chorégraphe Micheline Lelièvre portant sur les "corps en mouvement dans l'espace » en 2008, une étudiante, alors en première année DPLG ${ }^{6}$, écrivit : «Grâce à ces exercices, je me suis rendu compte de l'épaisseur de mon corps, de son organisation mais aussi de ses surfaces de contact, de ressenti. Ainsi, je sais aujourd'hui comment de simples gestes me permettent de faire travailler mon corps pour le rendre plus réceptif aux multiples contacts que nous avons avec les espaces qui nous entourent. [...] Il ne suffit pas de lire le site sur un plan mais il faut impérativement le vivre physiquement comme nous pourrons vivre et anticiper au fil des esquisses les façons d'utiliser notre aménagement. » Cet apport est fondamental car dans notre structure mentale occidentale, la séparation corps/esprit nous a plus ou moins éloignés de certaines évidences concernant la qualité des aménagements ainsi que leur pratique.

\section{Moments pédagogiques}

4 Par rapport aux deux niveaux indiqués en introduction et à leur fondement : il s'agit d'aider à une prise en compte de l'expérience vivante du sentir et de susciter des passages pour, d'une part, une relation préverbale dans un mode d'être immédiat et, d'autre part, des modes de description, une analyse, une mise en mots et en évocations plastiques et spatiales ainsi que des propositions de transformation destinées à autrui et à un monde commun. Pour cela, les principes sont les suivants : associer avec le plus de fluidité possible théorie et pratique, favoriser l'expérience, mettre en mouvement, laisser du temps, donner confiance, ne pas tout expliquer, revenir sur les expériences de séance en séance, d'année en année, avoir un canevas assez précis des phases (actions, gestes et outils pour quelles mises en mouvement) et ne pas tout prévoir. La plupart de ces principes se retrouvent d'une manière ou d'une autre en chacun de mes enseignements. Au semestre trois actuel (équivalence licence 2), j'associe directement le contenu d'un cours sur la représentation et l'expérience du paysage à des travaux dirigés intitulés " Construire l'espace »; en équivalence master 2 ou 3 , je relie un cours sur l'art dans l'espace public à l'encadrement d'un séminaire d'initiation à la recherche nécessitant une pratique située; entre les deux, dans une esquisse en début du semestre six (équivalence master 1) et dans une semaine intensive en fin du même semestre sur la spatialité, j'apporte des propositions d'outils afin que les étudiant.e.s trouvent leur chemin pour envisager le projet avec une approche ne dissociant pas le corps et l'esprit. Je vais maintenant présenter des moments pédagogiques correspondant à ces principes et à mon postulat.

\section{Disponibilité, s'y mettre}

5 Le moment pour une disponibilité est, bien sûr, ce qui amorce, ce qui permet de s'y mettre et qu'il y ait un développement. Grâce à la confiance sur laquelle je reviendrai, 
plusieurs temps se précisent. Le premier débute chaque séance et il revient au retour d'une pause, et si besoin quand la concentration s'échappe. Cette préparation est une sorte d'échauffement nécessaire pour aider à la concentration sans fixation, et pour que chacun.e trouve son rythme et son chemin pour s'y mettre. Si nous allons trop vite, sommes pressés par le temps, aucune relation élargie ne peut s'installer. Gestes d'automassage (de la tête aux pieds), savourer le silence, fermer les yeux, passer de l'écoute à l'entente, du regard fovéal à la vision périphérique, marcher sans but, penser à sa respiration apportent une temporalité favorable à une attention sans objet, à un rapport à soi, au groupe et au monde environnant dans une amplitude non focalisée. Donner du temps sans le calculer, ne rien attendre, laisser venir une fluidité en dehors des mots. Associant manière d'être, disposition sensible et mentale, ces commencements déconcertent parfois les étudiant.e.s qui ont l'habitude d'avoir des objectifs d'une certaine manière plus précis. Or, sans cette préparation, la perception (qui est toujours perception de quelque chose) domine sur le sentir, les habitudes et les protections créent un filtre entre les étudiant.e.s et ce qui leur arrive dans la simplicité de l'événement.

6 Le deuxième temps concerne la durée ainsi que la qualité d'attention : être disponible pour laisser venir ce qui arrive : à la fois ce qui est proposé dans le cadre d'un protocole expérientiel tout en se rendant disponible à soi-même et à l'environnement. Comme l'indique Micheline Lelièvre, intervenante régulière, se rendre disponible passe par " être présent à soi-même, à l'espace environnant, à ses perceptions propres ${ }^{7}$ ». Sarah Degraeve, danseuse intervenante depuis 2011, débute une séance de six heures consécutives en semestre trois par un temps au sol. Elle précise qu'avec ses propositions, «il va s'agir d'écoute, de disponibilité, d'ouverture. C'est tenter d'être présent à soi, aux autres et à son environnement grâce à un travail fin sur les perceptions. Je pars du corps et de ses perceptions : comment habiter son corps, voir, avoir conscience de soi, s'éveiller à soi = c'est sentir, ressentir et agir (notions que j'emprunte au $\mathrm{BMC}^{8}$ ) ». Ensuite, elle propose d'« habiter un espace, seul ou/et avec les autres = prendre conscience de sa verticalité et de son rapport à un horizon au travers de ses propres perceptions, et se déplacer ${ }^{9} »$. Je reviendrai sur l'horizon qui est l'autre relation primordiale et fondamentale associée au sol. Cette disponibilité est souvent vécue comme la redécouverte, évoquée en introduction, de sa présence incarnée, mais aussi du rapport au sol. Elle est utile aux étudiant.e.s pour reconsidérer leur approche, la prédominance de la vue par rapport aux autres sens, voire de se rendre compte de certains préjugés. Ainsi des étudiant.e.s en fin d'un enseignement en travaux dirigés disent qu'au début, mes indications leur avaient semblé « vagues » ou « abstraites ». Je ne leur demande pas d'abord de faire quelque chose, mais d'être disponibles, attentifs au monde environnant et de considérer des " éléments immatériels ». Par ailleurs, sans doute ne considèrent-ils (elles) pas tout ce qui est du côté de la géométrie, du calcul, des mesures ou des règles concernant le rendu spatial comme étant abstrait et correspondant à une conception ${ }^{10}$. C'est ainsi que, d'ailleurs, dans ce texte, je rencontre l'écueil de donner à comprendre un processus associant intimement mode d'être préverbal, corporéité, relation tonico-posturale, amplitude spatiale, relation aux horizons (interne, externe et temporel), aux poids et aux densités de relations. Or, comme l'écrit le philosophe Henri Van Lier (1959) : «Quand je jouis de cette prairie en pente, je ne l'organise nullement selon les courbes de niveau d'un cartographe ${ }^{11}$. " Celleci nous a d'abord sollicités, et donné envie de la dévaler en courant ou bien d'y rouler. Nous nous organisons pour ne pas tomber, nous testons le sol (s'il est glissant, mou ou 
dur, accidenté ou non), nous changeons nos appuis et nos repères ; corrélativement, "l'espace est impliqué dans notre motricité» (Maldiney, 1991, p. 216). Avec la disponibilité, il s'agit de porter son attention sur la manière dont nous nous organisons chacun.e en notre rencontre et contact avec le monde et autrui ainsi que sur ce qui se passe alors, comment l'espace lui-même se modifie. Seule l'expérience permet d'envisager que ce qui est corporel, ce qui est du côté d'un mode d'être, est très concret et concerne le poids, l'effort, la respiration, les relations tonico-posturales, la boucle oculomotrice. Il s'agit de l'éprouver. Les explorations proposées par Sarah Degraeve et, dans une autre séance de travaux dirigés, par l'artiste Jean-Christophe Nourisson, sont des traversées pour apprécier les organisations et transformations et ce, dans une certaine durée, en salle et en extérieur. Aider à différencier sentir et percevoir ne peut se faire sans s'y mettre et sans des moments de lâcher-prise; sans y être, il est impossible de saisir ce qui se passe et de reconsidérer les structures mentales héritées peu questionnées. À la fin de l'esquisse d'une semaine, une étudiante écrira : « Arpenter un terrain et être littéralement à son écoute peut amener à le saisir, et s'ancrer dans des réalités physiques relève du bon sens. » Elle se sera débarrassée de l'«attitude abstraite", qui, pour Francisco Varela, correspond au "scaphandre" mettant " habituellement à distance de son propre vécu » (Varela avec Thompson et Rosch, 1993, p 56).

7 Pour se rendre disponible à ces appréciations, différents outils sont proposés au fil des enseignements. Ainsi, lors d'une semaine intensive au semestre six, je fais fabriquer aux étudiant.e.s un sténopé en leur donnant deux consignes principales. La première est de le poser sous le nombril au niveau du bassin : au croisement de l'axe vertical et horizontal ${ }^{12}$ et en relation avec leur respiration. La deuxième est de se demander «comment la lumière traverse l'espace ». La technique du sténopé n'est pas d'abord proposée pour aboutir à des images mais pour son décalage par rapport à l'emploi habituel des appareils photos (il ne s'agit plus de viser quelque chose). Les consignes les aident à se situer dans et avec un environnement, être disponibles vis-à-vis de ce qui se passe et de faire partie du moment. Cela a lieu dans une temporalité et avec un élargissement à ce qui les entoure (quatre dimensions) qui n'est pas seulement défini par ce qui est strictement en face.

\section{Diversité, différences et pluralité}

8 Associée à la disponibilité en tant qu'accès à ce qui est éprouvé et qu'ouverture au monde, se situe la question essentielle de la relation à autrui. Elle se développe à trois niveaux : le premier est l'attention à ce qui est vécu par autrui (et donc à la manière d'y avoir accès), le deuxième est la capacité à travailler à plusieurs (et donc comment travailler avec des différences, une diversité et du commun) et le dernier est la destination de son projet à l'expérience d'autrui (comment se mettre à la place d'autrui).

9 "À partir de consignes simples que je donne, nous traversons ces différents temps d'explorations", indique Sarah Degraeve. Le pluriel importe. Il dit que ce moment d'exploration est réalisé ensemble ; il n'y a pas d'un côté l'intervenante et l'enseignante et de l'autre les étudiant.e.s. Par ailleurs, l'expérience est à la fois proprement personnelle tout en concernant d'autres personnes. En fin de séance, nous disant ce qu'ils (elles) ont traversé, les étudiant.e.s découvrent des différences et des éléments 
communs qui leur font envisager l'importance de la diversité ainsi que de ce qui est plus ou moins partagé. Cet échange leur permet de prendre confiance dans ce qui leur arrive (qui n'est donc pas illusoire) et de le relier à autrui. Trois semestres plus tard, lors de l'esquisse menée avec la paysagiste Françoise Crémel, nous faisons des groupes à mi-parcours, après deux jours d'approche et d'analyse personnelles du site d'intervention. À cette étape de la semaine, il s'agit de les aider à mobiliser leur compréhension issue de leur propre expérience et donc celle-ci comme apport d'analyse pour le projet. Dans la discussion qui clôture la semaine, les étudiant.e.s évoquent parfois un étonnement dans le fait d'avoir eu accès à autant de différences ; ils (elles) reviennent surtout à l'enrichissement qui les a amené.e.s à une reconsidération de leurs propres actions et relations aux espaces construits. Un étudiant écrira que «le travail de groupe est formateur [...] car il permet de voir comment une idée ou un concept peuvent être enrichis ou vus de différents points de vue suivant les sensibilités de chacun »; un autre relèvera que ceux-ci lui ont fait "comprendre [que] justement le projet doit être pensé pour tous ». L'importance décisive de l'échange de points de vue au sein du groupe constitué dans le processus de projet à destination d'autrui renvoie au deuxième niveau de sens indiqué en introduction. Ici se situe un passage délicat et formateur : celui du pour moi (chaque expérience est personnelle, c'est ce qui m'arrive), à quelque chose de partagé et d'établi à plusieurs afin que le projet soit pour autrui. Il s'agit d'aider les étudiant.e.s à différencier l'ego du soi ${ }^{13}$, pour que l'expérience subjective, au lieu d'être autocentrée, soit commerce ou conversation avec le monde; intersubjective, elle s'enrichit du travail en groupe, des discussions collectives et de la capacité de se mettre à la place des habitants. Une étudiante en 2017 relève avoir pu se placer « comme usager de l'espace [alors que] lorsque je conçois [...] il m'est difficile de prendre du recul ».

Pour Hannah Arendt, nous sommes «tous pareils, c'est-à-dire humains » et «jamais identiques » (Arendt, 1983, p. 41-43). La reconnaissance de la différence et de ce qui est partagé associe l'expérience (entre autres sensorielle) et le politique si l'on continue de la suivre quand elle écrit que «l'homme [...] n'existe qu'au pluriel» (Arendt, 1981, p. 117) et que la pluralité est «l'une des conditions existentielles fondamentales de la vie de l'homme sur terre » (ibid., p. 90). Celle-ci n'est pas abstraite, elle « est la loi de la terre $^{14} \gg$ (ibid., p. 34). Correspondant à notre coprésence (parfois difficile et tendue) ${ }^{15}$, elle induit, au-delà de la natalité, le fait que nous sommes les coconstructeurs (coconstructrices) de notre monde commun (toujours en cours) à partir de nos différents points de vue au sujet desquels nous avons à débattre. Une chose est de le dire, une autre est d'éprouver cette coconstruction, et de comprendre que cela participe à un commun au-delà des intérêts personnels.

11 Dans le séminaire d'initiation à la recherche, je l'aborde en favorisant des confrontations et des partages d'analyse de terrains et de situations ainsi qu'une attention portée à autrui, en particulier dans les entretiens, pour savoir écouter, laisser parler, se laisser bousculer par la parole d'autrui. Dans leur protocole d'investigation du terrain pour leur mémoire d'initiation à la recherche, deux étudiantes ont repris ou adapté l'outil du sténopé en tant que propre mise en disponibilité, présence au monde environnant et apport pour la compréhension des usages et pratiques. La première a " choisi le Polaroid pour ses clichés instantanés [...] qui permettent une analyse de l'espace public en termes de spatialité et [...] pour mettre en exergue [...] le rapport de l'individu à l'espace et aux autres » dans l'espace public. Surtout, il lui " permet de se concentrer et d'être dans l'espace (c'est-à-dire en relation avec ce qui [1]'entoure et 
avec les individus dans cet espace) ». La seconde a relevé l'importance de la prise en compte de la notion du « temps qui passe, de sa longueur et de son influence directe sur nous et nos sens [...] inhérente au sujet de la lumière nocturne. L'outil du sténopé [...] m'a permis de confirmer [...] trois temporalités lumineuses nocturnes. [...] J'ai pu [...] distinguer différents usagers [...] qui n'ont pas le même rapport à la lumière, à l'éclairage public et donc à l'espace public ». Alors que leur choix technique ne leur permettait pas un contrôle du rendu, elles ont mis en avant son intérêt pour le mode de relation et de saisie des choses. L'une "capte [ainsi] de manière plus vive ce qui se passe dans l'espace public » et pour l'autre, c'est «l'espace qui vient à nous et qui s'impose ».

12 En décalant les approches par rapport aux structures mentales habituelles (séparations corps/esprit, nature/culture, temps/espace, soi/monde), mon ambition pédagogique inclut l'engagement en tant que paysagiste et en tant que chercheur.e comme partie prenante de l'analyse et de la saisie d'une configuration spatiale et d'une situation concrète. Il serait contradictoire d'étudier la spatialité en mettant la sienne de côté et sans prendre en compte les différences, la diversité et la pluralité.

\section{Quitter l'espace pour la spatialité et l'horizon}

Que se passe-t-il au fur et à mesure de la journée avec Sarah Degraeve ? «Je les ai vus, dit-elle, s'imprégner de leurs propres sensations et de celles des autres. Je les ai vus entrer dans le langage du corps et du mouvement. » Avec ses consignes, elle les amène à « s'ouvrir, se relâcher (à ne pas confondre avec se relaxer) pour être dans l'écoute et la réalité du moment présent, des échanges qui sont en train de s'opérer. C'est recevoir ce que je vois, entends, touche et sens. J'ai vu leur propre transformation physique et celle de l'espace autour d'eux ». Relâchement et imprégnation sont deux indications essentielles qui prennent appui sur une disponibilité et se déploient avec une mise en confiance. Les transformations apportées concernent de manière indissociable un mode de présence au pluriel et l'espace environnant. Ici se situe la différence primordiale entre l'espace et la spatialité. Pour Erwin Straus, l'espace correspond à un système objectif et abstrait séparé du temps «sur lequel le sujet percevant promène sa visée à partir d'une position extra-mondaine » (Straus, 1989, p. 551). «La spatialité [n'est pas] synonyme d'espace géographique ou d'espace mathématique, [elle est indissociable de] l'individu [qui] fait l'expérience vécue de lui-même dans [et avec] l'espace ${ }^{16}$ » (ibid., p. 549). Les transformations vues et éprouvées par Sarah Degraeve, comme par les étudiant.e.s, concernent la spatialité. Vient alors la question politique : comment " vivre ensemble dans le monde » (Arendt, 1983, p. 92) ? Elle est articulée à la différence et à la pluralité qui mettent en avant l'espace entre, l'espace commun et l'horizon commun. Cette question rejoint celle du paysage si nous envisageons celui-ci comme étant en relation avec un horizon qui nous entoure à $360^{\circ}$. "Après tout, le monde est autour de moi, non devant moi » (Merleau-Ponty, 1986, p. 59). L'horizon, comme l'a écrit Michel Corajoud, correspond à l'échange et à l'épaisseur de contact entre le ciel et la terre et, de surcroît, nous sommes situés entre ces deux pôles. C'est pourquoi l'horizon est articulé à notre verticalité, qui, elle-même, est indissociable de notre motricité par la relation entre le proche et le lointain, le ici et le là-bas (sollicitation sensorimotrice, intentions). Le lien au paysage correspond à l'intention de l'artiste nord-américain George Trakas, dont les paroles et les réalisations nourrissent aussi l'imaginaire des étudiant.e.s. Avec ses œuvres, il « engage le corps, moment à moment, 
depuis les pieds, jambes, bassin, la colonne vertébrale, cœur, cou, bouche, nez, œil, oreille, cerveau, âme, de manière à mélanger les divers mouvements en une symphonie. [...] Je joue avec le corps comme un maître de ballet; je suis très sensible aux pieds : comment on sent le paysage avec le bassin» (Grout, 2007, p. 185). Cette dernière indication synthétise l'ensemble de cet engagement pour et avec le paysage qui est associé non pas (d'abord ou seulement) au regard mais à la relation sensorimotrice (aller vers et s'éloigner) à partir du contact avec le sol et dans le croisement des axes horizontal (bassin) et vertical (colonne vertébrale et posture érigée). L'élément central de ma pédagogie est une considération de l'horizon comme structure fondatrice de la relation au paysage comme spatialité. "L'espace du paysage » développé par Erwin Straus a un horizon à la différence de «l'espace géographique ».

La simple consigne de marcher donnée par Micheline Lelièvre lui permet d'inciter les étudiant.e.s à construire puis à moduler une relation à l'horizon. Envisager sa marche à plusieurs, en lien avec la marée, de face ou à reculons, faire le tour d'un bâtiment, apprécier les espacements, les interrelations, ce qui est caché à la vue, d'autres qualités de sol, toutes ces consignes induisent une mise en mouvement, une dynamique associant mode de présence, relation à autrui (connu et inconnu), au visible et à l'invisible, une non-dissociation du temps et de l'espace dans la relation du proche au lointain. Surtout, pour elle, chaque transformation correspond à une « manière de faire partie du monde ${ }^{17} »$.

\section{Confiance, lâcher-prise, ne pas savoir}

15 J'ai déjà évoqué ce moment du lâcher-prise associé à la disponibilité et à la confiance qui correspond au fait de changer ses outils, décentrer son approche, décaler ses gestes, quitter le regard fovéal pour la vision périphérique, ne pas prévoir, pour retrouver ce que Merleau-Ponty (1976, P. II) appelle le « contact naiff avec les choses ». Il ne peut pas y avoir de disponibilité sans confiance et celle-ci me concerne aussi. Après plus de quinze ans d'approfondissement de ce contenu dans mes travaux de recherche et grâce aux bilans partagés avec les étudiant.e.s, je relie autrement la théorie à la pratique, laissant la primauté à ce qui se passe dans le groupe et pour eux, tout en ayant un canevas séquencé. Une grande partie se déroule de manière préverbale. Par ailleurs, danseuses, chorégraphe et artistes $^{18}$ apportent leurs compétences pour amener les étudiant.e.s à traverser des expériences apparemment simples qui les transforment profondément. Cette confiance dans les enseignantes et les intervenant.e.s leur permet au fur et à mesure de ne pas se rigidifier et, sans abandonner complètement leur vigilance, de ne pas donner trop de poids à celle-ci ou de prévalence, pour laisser venir ce qui arrive. Alors commence une autre mise en confiance, celle qui correspond à ce que chacun.e traverse. Ce n'est d'ailleurs pas toujours aisé, tou.te.s les étudiant.e.s ne s'y mettent pas avec aisance et une fatigue vient. Il s'agit de les accompagner pour que chacun.e trouve ses appuis et son chemin. Avec Micheline Lelièvre, cela se développe avec « la perception du corps global, la relation gravité/contre-gravité, une perception fine du sol (avec les yeux fermés par exemple, ou en imaginant une autre qualité de texture), différents regards (passer du regard fovéal qui saisit un détail à la vision périphérique), ainsi qu'avec un aspect cognitif [lorsqu'elle propose des] distinctions entre le visuel, l'auditif et le kinesthésique ${ }^{19}{ }$ dans l'exploration des espaces. Avec cette présence à soi et au monde, il s'agit de retrouver «la confiance naturelle dans l'expérience sensorielle [ainsi que] sa validité » qui ont été abandonnées selon Erwin 
Straus « au profit d'une conscience désincarnée et sans monde [...] considérée comme allant de soi " (Straus, 1989, p.40). Cette "confiance naturelle", associée à une « connaissance pratique » (ibid.), polysensorielle, est un apport pour la compréhension $\mathrm{du}$ monde environnant ${ }^{20}$. Elle permet aux paysagistes d'appréhender l'être vivant présent et situé, ayant un certain poids et une sensation de pesanteur, étant relié à ce qui l'entoure et à autrui (et non en dehors du monde), en contact avec une qualité de sol. Une double difficulté ici est celle du passage à la fois de l'expérience à la saisie de ce qui s'est passé pour déduire des éléments de projet, et celle du caractère non intentionnel du sentir. Comme l'indique Straus, nous n'allons pas dans «l'espace du paysage » par notre volonté, ce mode d'être advient lorsque nous n'y prêtons pas attention. Pour cela, nous favorisons la vision périphérique qui participe au lâcherprise, à une ouverture à un espace englobant, aux mouvements et aux interrelations, ainsi que l'épreuve d'une présence qui dure sur place. Quand l'ennui vient, il n'y a plus rien à chercher (à percevoir, à stabiliser, à nommer). Nous les engageons aussi à apprécier l'importance des sons par rapport au visuel ${ }^{21}$. Par ailleurs, soit nous demandons qu'il n'y ait pas d'enregistrement, soit nous favorisons des modes de représentation qui ne les positionnent pas en face et en train d'observer comme s'ils (elles) étaient extérieur.e.s à une scène.

La paysagiste Soazig Friguel, diplômée en 2012, indique aujourd'hui :

« Je retiendrais qu'à travers ton enseignement, tu nous ( = ma promo) as sensibilisés à l'importance du corps dans la conception des espaces. À la fois notre propre corps qui regarde, qui analyse l'espace mais aussi notre corps en action qui "utilise" l'espace, qui l'occupe, l'investit, le change... (notion spectateur/acteur). C'est aussi regarder ce qui se passe, donc analyser les autres corps. C'est également nous faire prendre conscience que nous avons des émotions, des sentiments, des sensations, que nous ne sommes pas un corps neutre ou juste un corps quantifiable, et donc comment retranscrire le ressenti, le rapport à l'espace, aux choses, aux autres ${ }^{22}$.»

\section{Mettre en mots (passage 1)}

Nous avons vu qu'une disponibilité était indispensable pour se débarrasser de ses habitudes, c'est la première difficulté, la deuxième est celle du passage au langage. Comment dire ce que nous avons éprouvé et qui, avant cela, semblait si abstrait ? Pour cela deux moments se tissent ensemble, celui des échanges à l'oral qui nécessitent une écoute de ma part pour ne pas laisser filer ce qui commence à se dire, y compris par un questionnement, un doute ou un inconfort, et celui de l'écrit. Les discussions viennent en fin de séquence pour retraverser ce qui s'est passé et, nous l'avons vu, nous écouter les un.e.s les autres sans évaluation; de manière informelle, elles aident à évoquer une difficulté et surtout elles engagent la réflexion avec des retours à l'enseignement théorique mené en parallèle, ou à des ouvrages et à des textes parfois lus à haute voix.

Concernant l'écrit, je prévois plusieurs modalités. La première, dans les travaux dirigés et une semaine intensive, correspond à dix minutes finales pendant lesquelles je demande aux étudiant.e.s de répondre à une question. Ce moment réflexif apprécié leur permet de trouver leurs propres mots pour dire ce qui s'est passé et il leur évite une dilution. La mise en mots les amène à commencer à associer analyse et synthèse. La deuxième modalité se place en fin d'un enseignement et correspond à un des éléments du rendu évalué. Je demande à chacun.e de répondre, par exemple, à la question ouverte : "Qu'avez-vous appris pour le projet?» 
19 La troisième modalité est située maintenant au semestre six et elle fut mise au point par et avec Françoise Crémel. La mise en mots est traitée pédagogiquement dans l'association du sens (ce qui est dit après avoir été vécu et pas seulement observé), de la voix (présence au sens) et de l'espace (pouvoir dire aux autres de manière publique l'orientation du projet afin que le contenu ne soit pas creux et soit déjà une mise en mouvement des gestes pour le projet). Il s'agit de les amener à dire leur expérience, c'est-à-dire la spatialité straussienne, et donc à trouver les mots justes qui pourront porter leur intention de projet, lui donner toute sa cohérence et sa nécessité. Nous leur demandons d'assumer pleinement la force du langage comme expression d'un vécu et comme une manière de se porter dans le projet et d'engager celui-ci. Avec évidence, cela s'entend si le mot n'est pas approprié, si le sens n'y est pas. D'une part, la personne n'est pas convaincue et, d'autre part, nous ne le serons pas, et en particulier lors du rendu final. Ce moment lié au langage et à la présence fait partie de l'expérience, il nécessite là aussi un lâcher-prise ${ }^{23}$ pour aller au fond des choses avec le langage et prendre confiance. «J'ai apprécié l'exercice de concision qui nous était proposé, écrit une étudiante. Épurer nos phrases pour aller à l'essentiel. Si je donne sa justesse et sa force à un mot, pourquoi aurais-je besoin d'autres mots pour assurer la bonne réception de mon idée par mon interlocuteur ? [...] Dans le cadre du projet, il nous faut traduire nos réflexions afin de les rendre accessibles à autrui, qu'elles fassent sens. La justesse des moyens - le verbe en est un - est donc capitale. »

\section{Mémoire corporelle (passage 2)}

Avec la mise en mots, vient un deuxième passage de la spatialité au projet. Ce passage poursuit l'expérience vivante avec l'apport de la mémoire corporelle dans la saisie du site et/ou de la situation. Cet aspect de la mémoire corporelle est essentiel dans la méthode afin de trouver appui sur la matière de l'expérience. Une fois de plus il s'agit ici dans la pédagogie d'aider à ce qu'il y ait un lien (mais non causal, c'est bien toute la difficulté, parce que l'expérience est, comme je l'ai déjà écrit, " pour moi »). Revient alors la distinction entre le soi et l'ego, entre ce qui est l'expérience de l'anonyme (Merleau-Ponty) et ce qui est biographique, pour que la connaissance pratique soit celle d'un sujet situé dans le monde. « Habiter un espace, écrit Henri Maldiney (1991, p. 216), c'est être présent à lui et y être présent à soi... hors de soi-même." Ce double mouvement est au cœur du processus. Pour convoquer la mémoire corporelle, là aussi une disponibilité est nécessaire, une attention sans objet, afin de laisser venir des sensations (rétrécissement, enveloppement, enfermement, effet couloir, sensation d'allégement, envie de courir, de s'enfuir, de tâter le terrain, intensité, densité y compris de l'air ambiant, ne pas se sentir bienvenu). Au retour d'une présence sur une place publique, je demande aux étudiant.e.s de laisser émerger sur des grands formats, au mur ou au sol, ce qui leur revient de la spatialité, des diverses activités et de l'atmosphère du moment, des "éléments souvent absents des plans et des relevés ${ }^{24}$ " comme s'en souvient Soazig Friguel. Je leur demande de ne pas séparer le temps de l'espace et surtout de ne pas se situer en survol. À l'issue de ce processus, une étudiante indiquera "la libération de son geste et de sa pensée » pour la représentation d'un entre-deux (terre/ciel) qu'elle évoquera comme la mise en évidence de son envie de poursuivre ses études en paysage. 


\section{Sol commun}

Si j'insiste autant sur l'expérience c'est aussi parce que je trouve peu de lien concret et incarné, y compris dans les textes faisant partie d'une pensée venant de (ou nourrie de) phénoménologie (en dehors des textes de Merleau-Ponty), comme s'il y avait toujours les séquelles de la séparation corps/esprit, sujet/monde, temps/espace, avec lesquels je dois aussi composer moi-même. Dès lors, pour cette pédagogie, d'autres compétences que les miennes sont indispensables: principalement celles qui sont issues d'une compréhension de la relation entre le mouvement et les sens (y compris de la proprioception) venant de la danse et d'un imaginaire associé tel que la chorégraphie peut les susciter.

«La chorégraphie, écrit Micheline Lelièvre, a ceci de commun avec le travail du paysagiste qu'elle œuvre sur la construction d'espaces dans lesquels se meuvent des êtres, se tissent des liens entre des êtres et un environnement et que cela est destiné à autrui, ou conçu pour permettre à autrui de s'y mouvoir (en imagination éventuellement) $)^{25}$."

Si la considération du sentir est une expérience centrale, j'ai déjà évoqué deux difficultés le concernant. La première est que nous ne pouvons pas aller dans « l'espace du paysage » du fait de notre volonté ; il s'agit donc de lâcher-prise et de laisser-venir ce qui se fait dans une certaine durée, sans chercher quelque chose, pour privilégier un mode d'être et un horizon de sens. La deuxième est que ce mode d'être ne se traduit pas de manière causale dans une formulation de projet parce qu'il n'y a pas de détermination, d'où la nécessité des passages et de l'appel à la mémoire corporelle. Une troisième concerne la relation au politique qui, telle que je la pose, correspond au sentir comme mode d'être relié (être dans le monde et avec la pluralité) qui nécessite l'exploration d'un commun (de ce qui est commun ${ }^{26}$ ).

Dans l'expérience, le sol et l'horizon apportent le contact et la relation ayant les deux niveaux de sens présentés en introduction. Les étudiant.e.s seront amené.e.s à dialoguer avec le sol (terre, sable, béton, herbe, asphalte), en intérieur comme en extérieur, sur un plateau de scène à la Rose des Vents ${ }^{27}$ ou dans la rue.

«Ma proposition débute au sol, indique Sarah Degraeve, avec la prise de conscience de notre propre poids, se relâcher dans la terre, elle qui nous porte et nous soutient. Tout va naître de ce rapport que nous allons entretenir avec le sol. On ne le quittera plus, ce sera toujours de là que nous venons, sur lui que nous nous appuyons, grâce à lui que nous évoluons dans l'espace ensemble. Il nous donne sa réponse, nous lui donnons notre poids. Il nous accueille et nous permet de nous élever. Ce point de soutien, de référence et d'ancrage nous permet certainement d'établir ce lien étroit entre nos deux pratiques. »

Pour que ces indications nourrissent le projet, afin que le sol ne soit pas une surface inerte et abstraite, pour qu'il soit bien question de paysage et non d'un plan bidimensionnel sans horizon, il importe que le sol soit éprouvé, ainsi que sa propre relation au sol (la prairie en pente de Henri Van Lier), seul.e et avec autrui. Micheline Lelièvre, de son côté, incite à marcher, car le «mouvement est une ouverture à des possibles $^{28} »$; avec quelques indications, elle construit des situations qui engagent les étudiant.e.s à une présence au monde. Elle s'attache à déployer la relation à l'horizon comme une évidence et un projet demandant à être vécus. autrui, et non dans un espace abstrait. Pour un.e paysagiste cela voudra dire, d'une 
part, qu'une prise en compte du monde environnant, tel qu'il est éprouvé et tel qu'il (elle) s'y éprouve, l'assure d'une compréhension de ce qui l'entoure débarrassée de certains filtres, d'une mise à distance du réel et que, d'autre part, cela lui permet, à partir de l'existant, de projeter une transformation spatiale destinée à autrui. Dans l'introduction, j'indiquais le premier élément de mon postulat : le sentir étaye l'horizon politique d'un projet de paysage, dès lors, une présence au monde et à autrui (c'est-àdire qui n'est pas autocentrée) assure un fondement incarné au politique. Je pose, corrélativement que, d'une part, le politique pour un.e paysagiste débuterait dans le fait de projeter un sol commun destiné à la pluralité des êtres vivants, associé à un horizon spatial et temporel et que, d'autre part, une pensée du paysage ne séparant plus sujet et objet, nature et culture, corps et esprit, temps et espace participe au renouvellement du politique. Autrement dit, le sentir, en tant que mode d'être au monde du (et des) sujet(s) vivant(s), peut être considéré non seulement comme ayant une portée politique décisive, mais surtout comme étant ce qui porte le politique en tant qu'initiation d'un projet destiné au vivre-ensemble sur Terre.

\section{BIBLIOGRAPHIE}

Arendt, H., Condition de l'homme moderne (1958), traduit par G. Fradier, Paris, Calmann-Lévy, 1983.

Arendt, H., La Vie de l'esprit (1971), traduit par L. Lotringer, Paris, PUF, 1981.

Choay, F., Pour une anthropologie de l'espace, Paris, Éditions du Seuil, 2006.

Cross, C., Ludwig Binswanger, Chatou, Les Éditions de la Transparence, coll. « Philosophie », 2009.

Grout, C., Le Sentiment du monde. Expérience et projet de paysage, Bruxelles, La Lettre Volée, coll.

« Essais », 2017.

Grout, C., L'Horizon du sujet. De l'expérience au partage de l'espace, Bruxelles, La Lettre Volée, coll.

« Essais », 2012.

Grout, C., "Spatialité, situation, description », dans « Spatialité et situations », séminaire doctoral du LACTH, mis en ligne en 2014, URL : http://www.lille.archi.fr/ressources/20580/72/ lacth_crsemdoct5mars14_spatialit_.pdf.

Grout, C., «L'anonyme, à qui tout cela advient », dans Olive, J.-P. et Caullier, J. (dir.), Filigrane, $\mathrm{n}^{\circ}$ 9, L'individuel et le collectif dans l'art, Sampzon, Delatour France, 2009.

Grout, C., « Les axes en mouvement. Au sujet des œuvres de George Trakas », Les Carnets du paysage, $\mathrm{n}^{\circ} 13$ et $14,2007$.

Hall, E. T., La Dimension cachée (1966), Paris, Éditions du Seuil, coll. « Points », 2002.

Joseph, I., « Paysages urbains, choses publiques », Carnets du paysage, n 1, 1998.

Lesage, B., Jalons pour une pratique psychocorporelle. Structures, étayage, mouvement et relation, Toulouse, éditions Érès, 2000. 
Lussault M., L'Homme spatial. La construction sociale de l'espace humain, Paris, Éditions du Seuil, 2007, $\mathrm{p} 18$.

McCann, R. « "On the Hither Side of Depth": An Architectural Pedagogy of Engagement ", Environnemental and Architectural Phenomenology, 16, nº 3, 2005.

Maldiney, H. « La dimension du contact au regard du vivant et de l'existant » (1990), dans Penser l'homme et la folie, Grenoble, Éditions Jérôme Millon, coll. « Krisis », 1991.

Merleau-Ponty, M., Le Visible et l'Invisible (1964), Paris, Gallimard, coll. « Tel », 1991.

Merleau-Ponty, M., L'EFil et l'Esprit (1960), Paris, Gallimard, coll. « Folio essais », 1986.

Merleau-Ponty, M., Phénoménologie de la perception (1945), Paris, Gallimard, 1976.

Paulmier, C., «Lumières urbaines. Les enjeux de l'espace public nocturne », mémoire d'initiation à la recherche, ENSAPL, 2017.

Rouquet, O., La Tête aux pieds, Pantin, Recherche en mouvement, 1991.

Seamon, D., «A Way of Seeing People and Place : Phenomenology in Environment-Behavior Research », dans Wapner, S, Demick, J., Yamamoto, T. and Minami, H. (eds.), Theoretical Perspectives in Environment-Behavior Research, New York, Plenum, 2000.

Simondon, G., Cours sur la perception (1964-1965), Chatou, Les Éditions de la Transparence, 2006. Straus, E., Du sens des sens. Contribution à l'étude des fondements de la psychologie (1935), traduit par G. Thines et J.-P. Legrand, Grenoble, Éditions Jérôme Millon, coll. « Krisis », 1989.

Suard, L. « Un stade, un quartier, un territoire », mémoire d'initiation à la recherche, ENSAPL, 2017.

Tassin, E., Un monde commun. Pour une cosmo-politique des conflits, Paris, Éditions du Seuil, 2003.

Van Lier, H., Les Arts de l'espace, Bruxelles, Casterman, 1959.

Varela, F. avec Thompson, E. et Rosch, E, L'Inscription corporelle de l'esprit, sciences cognitives et expérience humaine, traduit par V. Havelange , Paris, Éditions du Seuil, coll. « La couleur des idées ", 1993.

\section{NOTES}

1. Le commun a ici un double sens, à la fois celui politique du commun qui est toujours à constituer et celui du monde commun au quotidien, de la vie commune. Pour l'un comme pour l'autre, un certain commun porte les actions (lui donne un horizon), qu'elles soient délibérément politiques ou citoyennes (par exemple la gestion des déchets ménagers qui dépasse l'aspect domestique). Le politique vient de la polis grecque qui diffère de la politique. Je renvoie aux textes de Hannah Arendt.

2. Le sens ici de "environnement» correspond à "monde environnant " pour Straus). Les travaux de Benoît Lesage du côté de la psychomotricité sont également des appuis précis et passionnants. En termes de méthode, je renvoie aux textes de Rachel McCann qui développe une approche fondée sur l'embodiement en tant que nécessité pour la conception architecturale et au texte du géographe David Seamon qui met en avant une approche phénoménologique.

3. Je souligne.

4. Une expérience située diffère d'une position qui, elle, est définie par des coordonnées géographiques en un point. Une situation inclut l'ouverture à la profondeur, une présence au monde environnant et des interrelations avec lui. 
5. Cela accompagne une analyse de toutes sortes de contraintes, de forces et de règles qu'il ne s'agit pas, bien sûr, de mettre de côté.

6. En 2008, la première année était alors celle après le concours d'entrée national, après deux ans d'enseignement supérieur.

7. Micheline Lelièvre, compte rendu des séances du laboratoire «Perception et construction de l'espace » en 2008.

8. "J'utilise le Body Mind Centering (BMC), pratique somatique, dans tout ce qui concerne le rapport fin au corps et à ses systèmes (squelettique, musculaire, osseux, organiques, les liquides...). [...] On peut, grâce à cet apport sensible, se sentir léger, lourd, se voir du point de vue du squelette et former des lignes grâce aux os et à ses articulations. On peut s'éveiller au volume de notre corps, nous percevoir et nous sentir de manière tridimensionnelle, grâce à un travail sur les organes. La peau elle aussi nous renseigne sur nos interactions avec le monde qui nous entoure."

9. Sarah Degraeve dans un échange de courriel avec l'auteure en décembre 2017. Les autres citations seront extraites de cet échange.

10. La psychanalyste et philosophe Caroline Gros explique: "Si une conception de l'espace géométrique s'est [...] imposée, c'est sa capacité à délimiter un espace visuel intégralement perceptible, intégralement divisible et intégralement homogène. Cette domination de l'intégralité incorporelle aux dépens de "l'incorporation des lieux" fait encore et toujours partie de notre appréhension spatiale du monde. » 2009, p. 247, souligné par l'auteure.

11. Sans pagination car source en ligne, je souligne.

12. J'associe le sténopé au projet oto date d'Akio Suzuki pour une relation auditive élargie et sans objet ; à son sujet, je renvoie à mon article «L'anonyme, à qui tout cela advient » (Grout, 2009, p. 191-211).

13. Le sentir correspond au mode d'être évoqué par Merleau-Ponty (1991, p. 254) quand le sujet est «l'anonyme enfoui dans le monde ». "Je, vraiment, c'est personne, c'est l'anonyme ; il faut qu'il soit ainsi, antérieur à toute objectivation, dénomination, pour être l'opérateur, ou celui à qui tout cela advient. » (Ibid., p. 299). D'une part, l'anonyme n'a pas de nom, pas de biographie et d'autre part, il n'est pas en surplomb ou en-dehors du monde, il est «celui à qui tout cela advient », celui qui est « avec » le monde pour Straus en une relation pré-verbale.

14. Souligné par l'auteur.

15. Cela amène à envisager non seulement la diversité des points de vue (qui fait aussi partie du moment politique dans l'horizon du vivre-ensemble) mais aussi les différences dans la vie quotidienne. Cet aspect de la diversité et du « pour tous » concerne une des tensions des espaces publics actuels à partir d'une différence vécue dans les relations spatiales et temporelles en raison de la mobilité, des déplacements voulus et subis, des rythmes parfois discordants, d'une absence de séjour ou de foyer.

16. La spatialité coïncide avec le sentir, ce que Straus thématise dans l'expression «avec le monde ». "Soi avec le monde, [...] L"'avec" implique que dans le sentir, je n'éprouve pas moimême et le monde par surcroît, mais que l'expérience vécue du sentir se déploie dans deux directions, vers le monde et vers le moi.» (Straus, 1989, p. 566). Le sens diffère donc de celui donné en d'autres disciplines, par exemple, pour le géographe Michel Lussault (2007, p. 18), la spatialité est « l'ensemble des usages de l'espace par les opérateurs sociaux ».

17. Micheline Lelièvre dans un courriel adressé à l'auteure en 2018.

18. George Trakas et, depuis quelques années, Jean-Christophe Nourisson.

19. Micheline Lelièvre, dans le texte déjà cité de 2008.

20. Maurice Merleau-Ponty: «Revenir aux choses mêmes c'est revenir à ce monde d'avant la connaissance dont la connaissance parle toujours et à l'égard duquel toute détermination est abstraite, signitive et dépendante, comme la géographie à l'égard du paysage où nous avons 
d'abord appris ce que c'est qu'une forêt, une prairie ou une rivière.» (1976, p III, souligné par l'auteur.)

21. Demander de délaisser la vue pour l'ouïe pose parfois des difficultés, en particulier aux étudiant.e.s qui disent être plutôt « visuel.le.s ».

22. Soazig Friguel, dans un courriel avec l'auteure en 2018.

23. Il demande plus ou moins de temps selon les étudiant.e.s. L'échauffement fait, ici aussi, partie du processus.

24. Soazig Friguel dans un courriel déjà cité.

25. Micheline Lelièvre dans un courriel adressé à l'auteure en janvier 2018.

26. Je n'aborde pas de front cette question dans les travaux dirigés, je l'évoque dans le séminaire d'initiation à la recherche et dans un cours en master.

27. Scène nationale voisine avec laquelle l'ENSAP de Lille a une convention depuis des années.

28. Micheline Lelièvre dans le courriel déjà cité, 2018.

\section{RÉSUMÉS}

Dans ce texte, je présente comment le sentir selon Erwin Straus (qui diffère du sensible et de la perception) porte potentiellement l'horizon politique d'un projet de paysage et comment ce postulat se traduit dans ma pédagogie en enseignement supérieur destinée à de futur.es paysagistes. Une question clé concerne la considération d'une qualité de présence à soi et au monde. Celle-ci permet, d'une part, de communiquer avec autrui à partir d'un sol commun en ayant un horizon et, d'autre part, de conforter une ouverture à la différence et à la pluralité. Par l'apport d'appuis concrets reliés à des références théoriques ainsi que des éléments de méthode (demandant essais et tâtonnements), les étudiant.es sont amené.es à distinguer sentir et perception. Alors les deux commencent à nourrir leurs choix de projet avec un horizon politique incarné. À partir de leurs textes et de moments pédagogiques, je présente les apports et les difficultés de cet enseignement mettant en avant la spatialité.

In this text, I explain how the notion of sensing (sensory experience ) according to Erwin Straus (which differs from the notions of sensation and perception) comprises the political perspective of a landscape project and how this postulate is applied in my teaching method for undergraduates in landscape architecture. A key question is the consideration of quality of presence in relation to oneself and the world. This makes it possible to communicate with other people on the basis of a common ground with a political horizon and supports the acceptance of difference and plurality. By means of concrete supporting elements linked to theoretical references and methods (requiring experimentation and trial and error) the students are encouraged to make the distinction between sensing and perception. These two notions then inspire their selected projects through an embodied political horizon. Based on their texts and classroom activities, I present the benefits and drawbacks inherent to this teaching method focusing on spatiality. 
INDEX

Mots-clés : expérience, spatialité, horizon, sol commun, politique

Keywords : experience, spatiality, horizon, common territory, politics

\section{AUTEUR}

\section{CATHERINE GROUT}

Catherine Grout est professeure d'esthétique HDR à l'ENSAPL, chercheuse au LACTH, ancienne résidente de la villa Kujoyama (1994-1995). Ses recherches portent sur le paysage, l'espace public, l'art, l'apparaître et l'expérience située à partir du sentir, de l'horizon et de la spatialité (Erwin Straus).

c-grout[at]lille.archi[dot]fr 